\title{
O uso do plano inclinado fixo na correção de mordida cruzada anterior dentária: relato de caso clínico
}

Use of fixed inclined plan for previous dental cross bite correction: clinical case report

uso del plan incluido incluido para la corrección anterior de mordida cruzada dental: reporte de caso clínico Tayane Silva Gonçalves VIANA ${ }^{1}$

José Henrique de Araújo $\mathbf{C R U Z}^{\mathbf{1}}$

Estefânia Queiroga de Santana e ALENCAR ${ }^{2}$

Fátima Roneiva Alves FONSECA ${ }^{2}$

Maria Carolina Bandeira MACENA ${ }^{2}$

${ }^{I}$ Cirurgiã(o) Dentista. Centro de Saúde e Tecnologia Rural, Universidade Federal de Campina Grande, UFCG 58708-110 Patos/PB, Brasil

${ }^{2}$ Professor(a) Doutor(a) do Curso de Graduação em Odontologia. Centro de Saúde e Tecnologia Rural, Universidade Federal de Campina Grande, UFCG 58708-110 Patos/PB, Brasil

\section{Resumo}

Introdução: Os tratamentos dos casos clínicos das más oclusões tem maiores índices de sucesso e facilidade de resolução quando são diagnosticadas precocemente na dentição decídua e mista. Objetivo: O propósito desse trabalho é relatar um caso clínico de mordida cruzada anterior dentária tratada com aparelho plano inclinado fixo. Relato do caso: Paciente do sexo masculino, 8 anos e 6 meses de idade, compareceu a Clínica Escola de Odontologia da UFCG diagnosticada com mordida cruzada anterior dentária e sob intervenção através do uso do plano inclinado fixo confeccionado na prórpia instituição, evidenciando as etapas de confecção desse método interceptativo. O aparelho foi confeccionado, instalado e utilizado em duas etapas de 15 dias cada na disciplina de Clínica Infantil II (Ortodontia). Conclusão: Os resultados obtidos foram satisfatórios, tendo um impacto corretivo da mordida cruzada anterior dentária, sem intercorrências e boa aceitação do paciente frente ao aparelho.

Descritores: Ortodontia Interceptora; Má Oclusão; Movimentação Dentária.

\section{Abstract}

Introduction: Treatments of clinical cases of malocclusions have higher success rates and ease of resolution when they are early diagnosed in deciduous and mixed dentition. Objective: The purpose of this paper is to report a clinical case of anterior dental crossbite treated with fixed inclined flat appliance. Case report: Male patient, 8 years and 6 months old, attended the UFCG School of Dentistry Clinic diagnosed with anterior dental crossbite and under intervention through the use of the fixed inclined plane made in the institution itself, showing the steps of making this interceptive method. The device was made, installed and used in two stages of 15 days each in the discipline of Children's Clinic II (Orthodontics). Conclusion: The results obtained were satisfactory, having a corrective impact of the anterior dental crossbite, uneventful and good patient acceptance in front of the device.

Descriptors: Orthodontics, Interceptive; Malocclusion; Tooth Movement.

\section{Resumen}

Introducción: Los tratamientos de casos clínicos de maloclusiones tienen mayores tasas de éxito y facilidad de resolución cuando se diagnostican temprano en dentición decidua y mixta. Objetivo: El objetivo de este trabajo es informar un caso clínico de mordida cruzada dental anterior tratada con un aparato plano inclinado fijo. Caso clínico: paciente masculino, de 8 años y 6 meses de edad, asistió a la Clínica de la Facultad de Odontología de UFCG diagnosticada con mordida cruzada dental anterior y bajo intervención mediante el uso del plano inclinado fijo realizado en la propia institución, que muestra los pasos de haciendo este método interceptivo. El dispositivo fue fabricado, instalado y utilizado en dos etapas de 15 días cada una en la disciplina de Children's Clinic II (Ortodoncia). Conclusión: Los resultados obtenidos fueron satisfactorios, con un impacto correctivo de la mordida cruzada dental anterior, sin incidentes y una buena aceptación del paciente frente al dispositivo.

Descriptores: Ortodoncia Interceptiva; Maloclusión ; Movimiento Dentario.

INTRODUÇÃO

O conhecimento da etiologia das más oclusões é essencial para o sucesso do tratamento ortodôntico, uma vez que um pré-requisito para a correção é a eliminação das causas. Percebe-se um crescente interesse no diagnóstico precoce e tratamento de má oclusões e a ênfase correspondente aos procedimentos preventivos e interceptores na determinação do tipo de tratamento mais adequado para cada caso ${ }^{1,2}$.

A má-oclusão constitui uma anomalia do desenvolvimento dentário e/ou dos arcos dentários, ocasionando problemas estéticos/funcionais, tendo como causas mais comuns as condições funcionais adquiridas. Entretanto, não se pode ignorar que o desenvolvimento osteogênico, a hereditariedade e o estado geral de saúde da criança são fatores que também contribuem para a instalação e/ou agravamento de má oclusões ${ }^{3}$.

O tratamento interceptador das más oclusões pode iniciar-se precocemente, sendo os processos biológicos que regulam o desenvolvimento da dentição decídua e mista os norteadores para a tomada de decisão. A correção em crianças deve ser iniciada mediante a necessidade, sendo importante recomendar o momento mais oportuno para realizálo. A ortodontia interceptora consiste no tratamento realizado nos estágios da dentadura decídua e mista, independentemente da gravidade do problema ou da mecânica envolvida ${ }^{4}$.

A mordida cruzada anterior afeta igualmente ambos os sexos, com prevalência que varia de 5,05\% a $18 \%^{5,6}$ representando de $19,3 \%$ a $53,2 \%$ do total das mordidas cruzadas ${ }^{7}$. Ela se caracteriza por uma relação sagital invertida entre os incisivos superiores e inferiores, e deve ser tratada antes que interfira no crescimento esquelético, podendo ser classificada em mordida cruzada anterior dental, funcional ou esquelética, todas com etiologias distintas, que servem para diferenciá-las. A mordida cruzada anterior dental ocorre devido a uma alteração na inclinação de um ou mais dentes, ou seja, dente superior posicionado por palatino com vestibuloversão do dente antagonista, estando a relação maxilomandibular correta. Além disso, o 
paciente apresenta relação molar de classe ${ }^{8,9}$.

Há controvérsias sobre quando começar o tratamento desse tipo de má-oclusão, de acordo com alguns autores a idade ideal é entre 8 e 11 anos, pois a raiz está sendo formada e o dente está no estado ativo de erupção ${ }^{10}$, enquanto outros autores defendem que quanto mais precoce for a desprogramação neural e muscular dos efeitos patológicos e consequente reabilitação dos sinais fisiológicos, menor será o tempo necessário para a correção da alteração da função do órgão (músculo/dente) ${ }^{11}$.

O tipo de aparelho a ser utilizado está na dependência da idade desses pacientes e da complexidade do caso. Nos casos de mordida cruzada anterior os aparelhos utilizados na correção podem ser fixos ou móveis e classificam-se segundo sua ação em passivos ou ativos. O Plano Inclinado Fixo é um aparelho de acrílico, fixo nos dentes anteriores, sua confecção é simples podendo ser realizada sobre o modelo de gesso do arco inferior ou diretamente na boca da criança em uma única sessão ${ }^{12}$. Atua como um plano guia anterior, aplicando uma pressão dirigida ligeiramente para vestibular sobre os dentes em relação de mordida cruzada. A pressão é exercida sempre que o paciente fecha a boca para mastigar ou deglutir. No momento da colocação do plano inclinado, observa-se uma abertura nos segmentos bucais posteriores ao ocluirr ${ }^{12,13}$.

Pode ser usado nos casos em que os incisivos superiores estão com mordida cruzada e com uma quantidade considerável de sobremordida vertical ${ }^{13}$.

A normalização da mordida cruzada anterior dentária com o uso do Plano Inclinado Fixo ocorre geralmente após duas ou três semanas, restabelecendo o contato oclusal posterior ${ }^{7}$.

Baseado na importância do diagnóstico e na interceptação precoce da mordida cruzada anterior, o presente trabalho objetiva relatar o caso clínico de paciente da Clínica Infantil de Ortodontia da Universidade Federal de Campina Grande (UFCG) em fase de dentição mista.

\section{CASO CLÍNICO}

Paciente do sexo masculino, 8 anos e 6 meses de idade, melanoderma, apresentou-se a clínica infantil de Odontologia da Universidade Federal de Campina Grande com queixa principal de "incisivos centrais superiores tortos". Após anamnese seguida de exame facial, físico extra e intraoral, observou-se que a criança encontrava-se em fase de dentição mista, terços faciais harmônicos, padrão dolicofacial, sem assimetrias evidentes. O paciente apresentava relação molar do tipo classe I de Angle. A partir das observações feitas nos exames clínicos e do diagnóstico de mordida cruzada anterior dentária (Figura 1), optou-se pelo tratamento com uso do Plano Inclinado Fixo (PIF) por ser um aparelho de baixo custo e que traz resultado rápido e eficaz.



Figura 1: Fotografia inicial: vista frontal.

A confecção do aparelho se deu de forma tradicional, inicialmente foi feita a moldagem da arcada inferior e superior do paciente com alginato tipo II (Dencrigel@) e confecção do aparelho em resina acrílica autopolimerizável (Orto-Clas $\left.{ }^{\circledR}\right)$ sobre modelo de gesso pedra tipo III (Herodent da Coltene(), respeitando as margens gengivais $\mathrm{e}$ usando para cada dente a ser descruzado pelo menos dois dentes inferiores. Após a demarcação no modelo de gesso dos dentes que iriam receber o aparelho, realizou-se o isolamento com isolante para gesso (Asfer®). Aglutinou-se o líquido ao pó da resina acrílica autopolimerizável e ao atingir a fase fibrilar fez-se uma massa redonda que é adaptada sobre o modelo de gesso. Com auxílio de uma lâmina de Cera 7 (Dentbras ${ }^{\circledR}$ ) recortada é feita a angulação de $45^{\circ}$. Na sessão seguinte o aparelho foi cimentado aos incisivos inferiores com cimento de ionômero de vidro VidrionC (SS White ${ }^{\circledR}$ ), foram feitas recomendações de uma alimentação mais líquido/pastosa e atenção com relação a higiene da área que recebeu o aparelho (Figuras 2 e 3 ).



Figura 2: Aparelho adaptado e instalado: vista frontal.

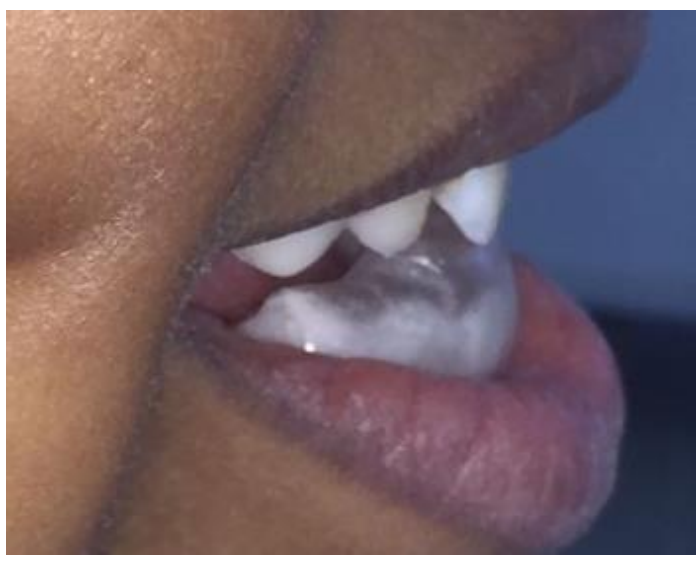

Figura 3: Aparelho adaptado e instalado. Vista lateral direita. 
O retorno foi marcado para sete e quinze dias após a cimentação. Na segunda avaliação o aparelho foi removido e observou-se uma oclusão anterior de topo (Figura 4).

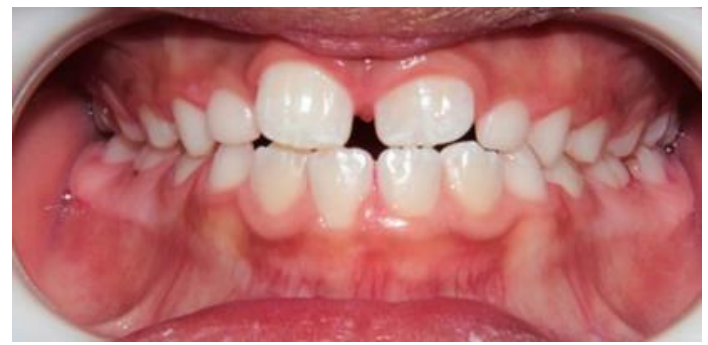

Figura 4: Oclusão após duas semanas do uso do aparelho. Mordida topo a topo.

Uma nova moldagem só foi feita após 2 meses da remoção do primeiro aparelho devido ao período de recesso da UFCG, então obteve-se um novo modelo de trabalho e um novo PIF seguindo a mesma técnica de confecção do primeiro aparelho (Figura 5). Este segundo aparelho foi cimentado (Figura 6) e após duas semanas de uso, o PIF foi removido e a mordida cruzada dentária anterior corrigida, porém como o paciente não apresentava ao início do tratamento um bom trespasse vertical, proservou-se a cada dois meses na clínica escola de Odontologia da UFCG (Figura 7).

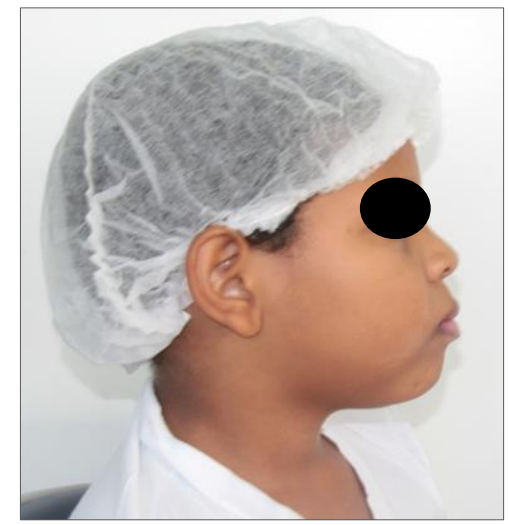

Figura 5: Perfil do paciente após uso do Plano Inclinado Fixo por 2 semanas.

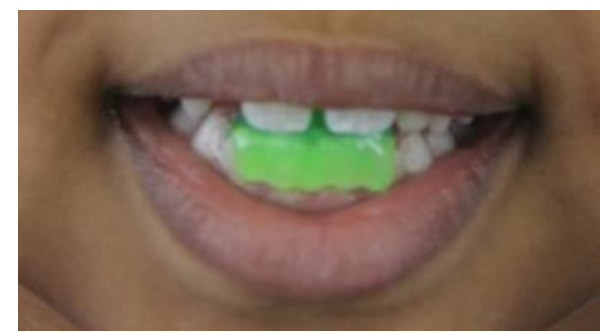

Figura 6: Novo aparelho instalado.

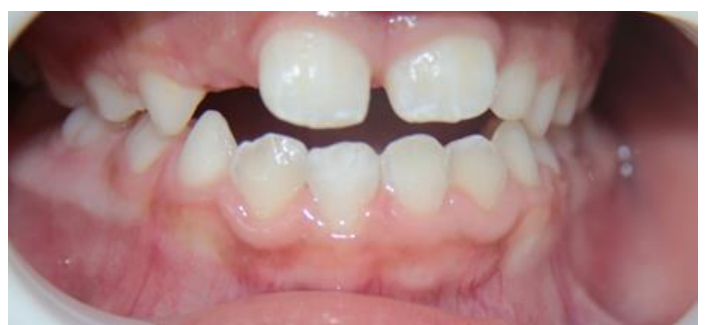

Figura 7: Mordida após mais duas semanas de uso. Observa-se ausência de trespasse vertical.
Após 4 meses da retirada do PIF o paciente retornou e pode-se observar estabilidade na correção da mordida cruzada assim como correção no sentido vertical com uma quantidade considerável de sobremordida (Figura 8 e 9).



Figura 8: Estabilidade na correção da mordida após 4 meses. Vista frontal.

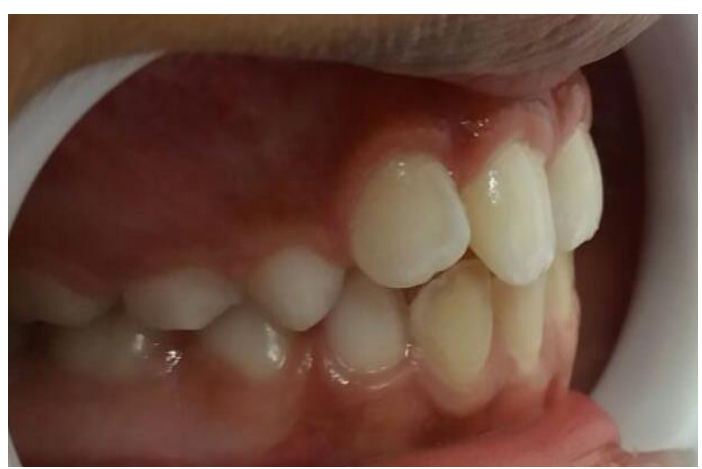

Figura 9: Estabilidade na correção da mordida após 4 meses. Vista lateral direita.

\section{DISCUSSÃO}

Quando a mordida cruzada anterior ocorre nas fases das dentaduras decídua ou mista, é coerente a indicação de tratamento precoce, uma vez que a mesma pode causar desgastes na face vestibular dos dentes superiores envolvidos, recessões gengivais nos incisivos inferiores, além de alterar o crescimento normal dos maxilares no sentido ântero-posterior ${ }^{11}$.

A identificação e resolução dos fatores causais envolvidos, juntamente com o diagnóstico diferencial entre uma condição dentária, funcional ou esquelética, são de suma importância para o êxito do tratamento da mordida cruzada anterior ${ }^{6,14}$.

No caso clínico relatado, com o paciente em relação cêntrica, foi observada alteração na inclinação dos dois incisivos superiores causando cruzamento anterior e relação molar de classe I de Angle, fatores que apontam para uma mordida cruzadas anterior dentária. $\mathrm{O}$ fato de o paciente se encontrar numa fase de dentição mista precoce apontam para a escolha de tratamentos como aparelhos removíveis com mola digital, plano inclinado fixo em resina acrílica, lâmina de madeira entre outros ${ }^{2,15-16}$.

Devido as características da má oclusão do caso optou-se por um pano inclinado fixo uma vez que o aparelho fixo possui maior poder de ação, liberando uma força contínua, quando comparado aos removíveis, além de permitir o controle tridimensional do dente a ser movido ${ }^{17-18}$. Este 
aparelho se comporta como uma extensão das bordas de contato dos incisivos inferiores nas faces linguais dos dentes superiores, então no movimento de fechamento da boca, a mandíbula é forçada a se posicionar mais para posterior, e os dentes superiores que estavam palatinizados serão movidos para vestibular ${ }^{19}$.

A ausência de trespasse vertical inicial no caso apresentado foi um fator de confundimento ao uso do plano inclinado fixo porém, diante de todas as demais características do caso, da facilidade de confecção do aparelho e da possibilidade de ajuste rápido da má oclusão optou-se por este modelo de tratamento. Também levou-se em consideração o fato de se tratar de um serviço público, sendo este aparelho viavél por ser de baixo custo e grande eficiência ${ }^{9,20}$.

As vantagens do PIF residem na facilidade de fabricação, simplicidade de ação, agilidade no prazo de correção, e, além disso, leva vantagem sob os aparelhos ativos que necessitam da completa erupção dos dentes para sua adaptação, enquanto que para o PIF uma erupção parcial é suficiente ${ }^{21}$. É possível observar leve desconforto, dificuldade de alimentação e higienização como desvantagens do uso desse aparelho sendo, portanto, importante que seja feito uma orientação em relação a uma alimentação mais pastosa e reforço da higienização, todavia, no presente caso clínico não foi relatado nenhum desconforto por parte do paciente.

Durante a confecção do PIF é de suma importância que seja feito com laminade cera 7 um ângulo de $45^{\circ}$ com o longo eixo dos incisivos inferiores, a fim de que não haja resultante vetorial em favor da intrusão do dente cruzado, sendo igualmente importante que haja espaço no arco superior para que ocorra o descruzamento da mordida. Além disso, o recorte do acrílico deve respeitar as margens gengivais para evitar a inflamação do tecido periodontal ${ }^{9}$. No caso clínico relatado, como o paciente já não possuía um trespasse vertical adequado, a inclinação $45^{\circ}$ graus foi rigorosamente testada para que não ocorresse uma intrusão dentária e diminuísse ainda mais este trespasse vertical.

Mesmo com todos os parâmetros respeitados pode-se observar que ao final da segunda fase do tratamento o paciente encontrou-se com ausência de trespasse vertical, porém em se tratando de paciente na fase dentição mista intertransicional, o protocolo foi aguardar a erupção natural destes elementos. Após 4 meses da retirada do segundo PIF confeccionado, o trespasse vertical foi normalizado.

Deste modo, o PIF pode ser considerado como um aparelho importante na fase de intercpectação do problema, como também age de forma preventiva permitindo um desenvolvimento harmônico do aparelho mastigatório, melhor repercutindo na qualidade de vida do paciente ${ }^{9,15}$.

Alternativamente ao que foi proposto no presente caso clínico, Bravo e Culca ${ }^{22}$ relataram um caso de uma menina de 8 anos em que seu sorriso a incomodava por ter dentes tortos. Ele apresentou uma má oclusão Classe I com mordida dentária anterior entre os elementos 11/41 e 21/31 acompanhada de falta de espaço para a erupção do elemento 15. Das possibilidades de tratamento, optaram por uma placa Hawley ativa com parafuso expansão anterior e lateral. Finalmente, a eficácia do tratamento de Placa ativa de Hawley quando a mordida cruzada anterior é combinada e a recuperação do espaço para a erupção do dente, permitindo uma orientação correta oclusal no paciente.

Portanto, comparativamente ao presente caso clínico, é possível tratar uma má oclusão de maneiras diferentes e obter o mesmo resultado.

CONCLUSÃO

A mordida cruzada anterior dentária se diagnosticada precocemente e com tratamento adequado tem rápida resolução e bom prognóstico, que para esse tipo de má oclusão o plano inclinado fixo o aparelho ortopédico mais indicado. Esse aparelho, além do fator custo/benefício é bem indicado para pacientes não colaboradores e é de fácil confecçãose tornando também uma opção para o sistema público de saúde uma vez que, não necessitaria de recursos de alto custo e por ser indicado para uma má oclusão de fácil diagnóstico.

\section{REFERÊNCIAS}

1. Macena MC, Katz CR, Rosenblatt A. Prevalence of a posterior crossbite and sucking habits in Brazilian children aged 18-59 months. Eur J Orthod. 2009;31(4):357-61.

2. Tashima AY, Verrastro AP, Ferreira SLM, Wanderley MT, Guedes-Pinto E. Tratamento ortodôntico precoce da mordida cruzada anterior e posterior: relato de caso clínico. JBP j bras odontopediatr dontol bebê. 2003;6(29):24-31.

3. Thomaz EBAF, Valença AMG. Prevalência de má-oclusão e fatores relacionados à sua ocorrência em pré-escolares da cidade de São Luís - MA - Brasil. RPG Rev Pós Grad 2005; 12(2):212-21

4. Silva-Filho og. Protocolo de tratamento em duas fases: definindo conceitos. In: ortodontia interceptativa: protocolo de tratamento em duas fases. São Paulo: Artes Médicas; 2013.

5. Bittencourt MAV, Machado AW. An overview of the prevalence of malocclusion in 6 to 10 -year-old children in Brazil. Dental Press J Ortod. 2010;15(6):113-22.

6. Almeida RR, Oliveira GF, Almeida MR, Conti AC, Navarro RL, Oltramari-Navarro PV. Correção da mordida cruzada anterior na dentadura mista com aparelhos fixos. Rev Clin 
Ortod Dental Press 2010;9(5):86-100.

7. Moruzzi CD, Gumieiro EH, Pequeneza RAP, Almeida RC. Correção de mordida cruzada anterior com plano inclinado fixo. Ortodontia. 2013;46(4):381-89.

8. Bayrak S, Tunc ES. Treatment of anterior dental crossbite using bonded resin-composite slopes: case reports. Eur J Dent. 2008;2(4):303-6.

9. Figueiredo PBA, Ferraz RP, Silva VC, Junior JMP, Silva ARQ, Silva AI. Plano inclinado no tratamento da mordida cruzada anterior: relato de caso clínico. RFO UPF. 2014;19(2):229-233.

10. Prakash P, Durgesh BH. Anterior crossbite correction in early mixed dentition period using catlan's appliance: a case report. ISRN Dent. 2011;2011:298931.

11. Rossi LB, Pizzol KEDC, Boeck EM, Lunardi N, Garbin AJI. Correção de Mordida Cruzada Anterior Funcional Com a Terapia de Pistas Diretas Planas: Relato de Caso. FOL Faculdade de Odontologia de Lins/Unimep. 2012;22(2):45-50.

12. Forjaz MB, Osório MDSC. Mordidas cruzadas nas dentições decíduas e mistas [monografia]. Piracicaba: Faculdade de Odontologia de Piracicaba, Universidade Estadual de Campinas UNICAMP; 1995.

13. Jirgensone I, Liepa A, Abeltins A. Anterior crossbite correction in primary and mixed dentition with removable inclined plane (Bruckl appliance). Stomatologija. 2008;10(4):140-4.

14. Fernandes LB, Marsillac MW, Cariello A. Mordida cruzada anterior causada por dentes supranumerários. Rev Gau Odontol. 2005;53(3):247-50.

15. Künh A, Bringhenti A, Perussolo B. Tratamento alternativo para mordida cruzada anterior. RFO UPF. 2001;6(1):27-31.

16. Martins MM, Almeida MAO. Mordida Cruzada. In: Almeida MAO, Quintão CCA, Jr Capelli J. Ortodontia: Fundamentos e Aplicações Clínicas. Rio de Janeiro: Guanabara Koogan; 2008.

17. Tanaka OM, Maciel JVB, Kreia TB, Ávila ALR, Pithon MM. The Anterior Cross-bite: the paradigm of interception in orthodontics. Rev Clín Pesq Odontol. 2010;6(1):71-8.

18. Chibinski ACR, Czlusniak GD, Melo MD. Pistas Diretas Planas: terapia ortopédica para correção de mordida cruzada funcional. R Clin Ortodon Dental Press. 2005;4(3):64-72.

19. Woitchunas DR, Busato C, Tocheto LR, Brockstedt RH. Mordidas cruzadas anteriores: diagnóstico e tratamento da pseudoclasse IIIrelato de um caso clínico. RFO UPF. 2001; 6(2):23-8

20. Sari S, Gokalp H, Aras S. Correction of anterior dental crossbite with composite as an inclined plane. Int J Paediatr Dent. 2001;11(3):201-8.
21. Macdonald M, Avery DJ. Odontopediatria para crianças e adolescentes. São Paulo: Elsevier; 2011.

22. Bravo JJLC, Culca FAO. Mordida cruzada anterior dentaria en denticion mixta [monografia]. Chimbote - Perú: Facultad de Ciencias de la Salud Escuela Profesional de Odontología Segunda Especialidad en Odontopediatría; 2018.

\section{CONFLITO DE INTERESSES}

Os autores declaram não haver conflitos de interesse

\section{AUTOR PARA CORRESPONDÊNCIA}

\section{José Henrique de Araújo Cruz \\ Rua Paulo Diogenes, 57 - Bairro Centro, 59990000. Rafael Fernandes - RN, Brasil Telefone: (83) 99625-0125 \\ E-mail: henrique_araujo1992@hotmail.com}

Submetido em 07/11/2019 Aceito em 22/10/2020 